\title{
Evaluación Biomecánica del Efecto de Bisfosfonatos y Aceite de Oliva en un Modelo de Remodelación Óseo en Fémur de Rata. Efectos en la Remodelación Ósea
}

\author{
Biomechanical Evaluation of the Effect of Bisphosphonates and Olive Oil in \\ a Model of Bone Remodeling in Rat Femur. Effects on Bone Remodeling
}

\author{
Escudero, C.. ; Virga, C. ${ }^{1}$; Aguzzi, A. ${ }^{\text {; }}$ Aramburu ,G. ${ }^{1}$ \& De Leonardi, A. ${ }^{1}$
}

ESCUDERO, C.; VIRGA, C.; AGUZZI, A.; ARAMBURU, G. \& DE LEONARDI, A. Evaluación biomecánica del efecto de bisfosfonatos y aceite de oliva en un modelo de remodelación óseo en fémur de rata. Efectos en la remodelación ósea. Int. J. Odontostomat., 11(1):19-24, 2017.

RESUMEN: Estudios han demostrado que el aceite de oliva $(\mathrm{O})$ con sus compuestos fenólicos tienen efectos positivos en diversos biomarcadores fisiológicos. Análisis previos han demostrado que los bisfosfonatos, son potentes inhibidores de la resorción ósea. Estudiar el efecto del tratamiento combinado de alendronato $(\mathrm{AL})$ y pamidronato $(\mathrm{PA})$ y de $\mathrm{O}$ sobre la regeneración ósea. Las fórmulas se dosificaron $0,5 \mathrm{mg} / \mathrm{kg}$ de peso para $\mathrm{AL}$, y de 0,6 mg/kg de peso para PA. El O se administró en la dieta, $50 \mathrm{~g} / \mathrm{Kg}$. Cincuenta y cuatro ratas macho de la línea Wistar se dividieron en 6 grupos. El grupo control (C), recibió semanalmente $0,3 \mathrm{ml} / 100 \mathrm{~g}$ de solución salina vía subcutánea. El grupo (AL) recibió semanalmente por vía subcutánea en el miembro posterior izquierdo. El grupo (PA) se colocó igual que el grupo anterior. El grupo (O) fue tratado en la alimentación y en las áreas de la cirugía recibieron inyección subcutánea con solución fisiológica. El grupo (ALO) recibió tratamiento combinado con $\mathrm{AL}$ y O. El grupo (PAO) se trató igual al anterior. Se obtuvieron muestras de fémur en tiempos 15, 30, 60 y 90 días, que se incluyeron en solución fisiológica y mantenidos a $-20^{\circ} \mathrm{C}$. Los estudios estadísticos se realizaron a través del análisis de la variancia a dos y tres criterios de clasificación. Sólo el factor días influye significativamente sobre los valores. Las diferencias entre drogas no resultaron estadísticamente significativas. Tampoco se verificó interacción significativa entre los factores Droga y etapa. Los valores más elevados de fuerza de ruptura aplicada, se registraron a los 90 días. No se encontraron diferencias significativas en los ensayos biomecánicos, poniendo de manifiesto la acción sistémica de los fármacos. Estas acciones fueron benéficas al aumentar la rigidez.

PALABRAS CLAVE: bisfosfonatos, aceite de oliva, ensayos biomecánicos.

\section{INTRODUCCIÓN}

La mecánica y la ciencia de materiales estudian los efectos y la relación entre las fuerzas aplicadas sobre una estructura o cuerpo rígido y la deformación producida (Buehler, 2008). El hueso, para su estudio, se puede considerar tanto un tejido como una estructura, ya que desempeña dos funciones básicas: control del metabolismo de $\mathrm{Ca}, \mathrm{P}$ y Mg (función fisiológica) y soporte del organismo y protección de órganos (función mecánica). La complejidad mecánica del tejido óseo, compuesto de hueso cortical y hueso trabecular, ambos con comportamientos mecánicos distintos, supera la de la mayoría de los materiales utilizados en ingeniería (Eppell et al., 2006).
Para mejorar los tratamientos aplicados contra enfermedades osteodegenerativas, como es el caso de la osteoporosis, resulta imprescindible optimizar las técnicas de diagnóstico que se basan principalmente en establecer correlaciones entre las variables biomecánicas y las distintas variables que proporciona el análisis de la cantidad y la calidad ósea (Guede et al., 2010).

Los bifosfonatos constituyen un grupo de fármacos inhibidores de la resorción ósea, utilizados en el tratamiento de numerosas patologías como la osteoporosis, la enfermedad de Paget, el mieloma

\footnotetext{
${ }^{1}$ Cátedra de Farmacología y Terapéutica. Departamento de Patología Oral, Facultad de Odontología. Universidad Nacional de Córdoba, Cordoba, Argentina.
} 
ESCUDERO, C.; VIRGA, C.; AGUZZI, A.; ARAMBURU, G. \& DE LEONARDI, A. Evaluación biomecánica del efecto de bisfosfonatos y aceite de oliva en un modelo de remodelación óseo en fémur de rata. Efectos en la remodelación ósea. Int. J. Odontostomat., 11(1):19-24, 2017.

múltiple, la hipercalcemia maligna y las metástasis óseas asociadas al cáncer de mama o de próstata (Fuchs et al., 2007). El principal efecto farmacológico de los bifosfonatos es la inhibición de la resorción ósea, mediante una disminución de la actividad de los osteoclastos, sin intervenir en la formación y mineralización del hueso. Son fármacos utilizados a nivel mundial con unos claros beneficios contrastados clínicamente. $A L$, produce incrementos en la densidad mineral ósea que se pueden observar transcurridos tan sólo 6 meses del inicio de la terapia. PA, Es uno de los más potentes y se emplea para disminuir el dolor producido por las metástasis óseas asociadas al cáncer de mama y al mieloma múltiple (Giavaresi et al., 2001).

Por otro lado, en los últimos años se han producido numerosas investigaciones que concluyen en que una interesante manera de prevenir la pérdida ósea sería a través de los polifenoles presentes en el $(0)$ extra virgen (Cicerale et al., 2010). El (O) se compone principalmente de los triacilgliceroles (triglicéridos o grasas) y contiene pequeñas cantidades de ácidos grasos libres (FFA), glicerol, fosfátidos, pigmentos, compuestos de sabor, esteroles, y partículas microscópicas de oliva. Los triacilgliceroles son la principal reserva de energía para las plantas y los animales (Madrona et al., 2009).

El objetivo de esta investigación es el de estudiar el efecto de la administración combinada de bisfosfonatos por vía subcutánea y de (O) por vía oral, sobre la calidad de hueso posterior a la regeneración tisular de cavidades óseas neoformadas como alternativa terapéutica para dar respuesta a muchas situaciones clínicas en odontología.

\section{MATERIAL Y METODO}

Preparación de las formulaciones: Se prepararon soluciones para ser aplicados por vía subcutánea. La fórmula farmacéutica con AL se preparó con una dosificación de 0,5 mg/Kg de peso corporal, y la de PA con una dosificación de $0,6 \mathrm{mg} / \mathrm{Kg}$ de peso corporal. El O se administró junto con la dieta, $50 \mathrm{~g} / \mathrm{Kg}$ de comida.

Preparación de los animales de experimentación: Se obtuvieron las muestras de fémur de ratas machos Wistar luego de realizar una cirugía en las tibias de los animales. Previamente fueron anestesiados con una solución de ketamina/xilazina en relación $8 \mathrm{mg} / 1,28 \mathrm{mg}$ respectivamente por cada
$100 \mathrm{~g}$ de peso corporal. Previa asepsia del campo quirúrgico con yodopovidona. Se realizó con bisturí Bard Parker y hoja $N^{\circ} 15$ una incisión longitudinal en ambas tibias y se procedió al decolado hasta llegar a exponer el hueso. Con una fresa número 6 y a rotación manual para no producir necrosis ósea, se realizó una cavidad en la parte plana de cada tibia hasta llegar al hueso medular. Dicha cavidad no fue rellenada con ningún material y sólo será reparada por su propio coágulo. Luego de realizada las intervención quirúrgica, se recolocaron los planos en posición y se suturó la herida con hilo reabsorbible.

Los animales se dividieron aleatoriamente en 6 grupos de 9 ratas cada uno, para la prueba de las drogas y fueron organizados de la siguiente manera: Un grupo fue grupo control (C). Los animales de este grupo recibieron semanalmente $0,3 \mathrm{ml} / 100 \mathrm{~g}$ de peso corporal de solución salina por vía subcutánea cercana a la intervención quirúrgica y se administró agua corriente de red como agua de bebida. El segundo grupo recibió (AL) por vía subcutánea profunda en el miembro posterior izquierdo cercano a la zona quirúrgica, y en el miembro posterior derecho se le aplicó solución salina como el grupo control. El tercer grupo (PA) se le administró semanalmente la droga por vía subcutánea profunda en el miembro posterior izquierdo de igual manera que el grupo anterior. El cuarto grupo $(\mathrm{O})$, recibirá tratamiento con $\mathrm{O}$, siendo alimentados ad libitum, con una ración de $40 \mathrm{~g}$ día/rata, al que se le adicionó previamente el principio activo durante el tiempo que duró el experimento y en las áreas de las cirugías recibieron inyección subcutánea de solución salina como el grupo control. El quinto grupo de tratamiento se administró en forma combinada $\mathrm{AL}$ subcutáneo y ADO por vía oral en la dieta (AL+ADO). El sexto grupo de tratamiento combinado PA subcutáneo y ADO por vía oral en la dieta (PA+ADO)

Los animales se mantuvieron en bioterio en jaulas colectivas con alimento balanceado y agua de bebida ad libitum, a una temperatura de $22-26^{\circ} \mathrm{C}$, con un ciclo luz-oscuridad: $12 \mathrm{~h}-12 \mathrm{~h}$ durante el tiempo que duró el experimento. Se monitoreó a los animales cada tercer día, verificando su estado de salud general y el miembro sometido a experimentación en particular. El manejo de los animales es realizado siguiendo los lineamientos del "Nacional Institute of Health" $(\mathrm{NIH})$, especificaciones técnicas para la producción, cuidado y uso de los animales de laboratorio. Al finalizar el experimento se sometieron a eutanasia los animales mediante inyección intracardíaca de cloruro de potasio, bajo anestesia general. 
Los sacrificios para la toma de muestras fueron a los $0,7,15,30,60$ y 90 días.

Estudios biomecánicos: Los fémures fueron resecados, se disecaron los tejidos blandos y se prepararon para los ensayos destructivos de flexión a tres puntos, los cuales fueron conservados en solución fisiológica y mantenidos a $-32^{\circ} \mathrm{C}$. Los estudios fueron realizados con apoyo de personal del Laboratorio de Biología Ósea y Metabolismo Mineral de Rosario, Argentina. Se utilizó un equipo Instron 4411 (Instron Corp, Norwood, MA, USA) para medida de carga de fractura en un ensayo a tres puntos Los puntos de apoyo se colocaron a $11 \mathrm{~mm}$ y la fuerza vertical aplicada entre ellos se realizó a una velocidad de $0,254 \mathrm{~mm} / \mathrm{s}$, registrándose carga máxima $(\mathrm{N})$ parámetro que será utilizado para evaluar la resistencia ósea. Este parámetro fue utilizado como medida del efecto sistémico producida por las drogas utilizadas.

Estudios estadísticos: Con base en el tamaño de la muestra y asumiendo no-normalidad de los datos se decidió realizar el análisis estadístico con pruebas estadísticas no paramétricas. La prueba de Kruskal-Wallis es útil para comparar tres o más grupos, por lo que se empleó inicialmente para saber si existían diferencias entre los grupos. Se consideró diferencias significativas si $(p>0,05)$. Este estudio estadístico planteado además permitió determinar si existe asociación entre los efectos de los tratamientos y el tiempo. Para el análisis de datos, construcción de gráficas y análisis estadísticos se utilizó el software (GraphPad Prism 2.0. GraphPad Software, San Diego, CA USA).

\section{RESULTADOS}

Ensayo de flexión: Si la carga u fuerza aplicada se incrementa, el tejido responde con un ligero incremento de esfuerzo, acompañado de un aumento de deformación. Si se continúa aumentando la carga a flexión, el hueso comenzará a ceder y finalmente se alcanza el punto de ruptura o fractura. El área bajo la curva del gráfico fuerza-deflexión, representa la energía que absorbió el hueso antes de la falla. (Fig. 1)

Tenacidad o energía absorbida: De acuerdo al resultado del test de ANOVA, sólo el factor etapa o días influye significativamente sobre los valores de Tenacidad o energía absorbida $(p<0,05)$. Las diferencias

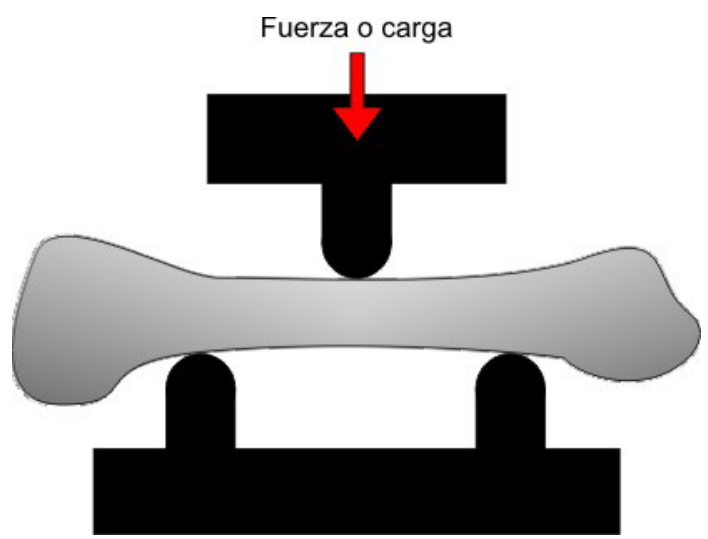

Fig. 1. Esquema de ensayo de flexión a 3 puntos sobre hueso largo.

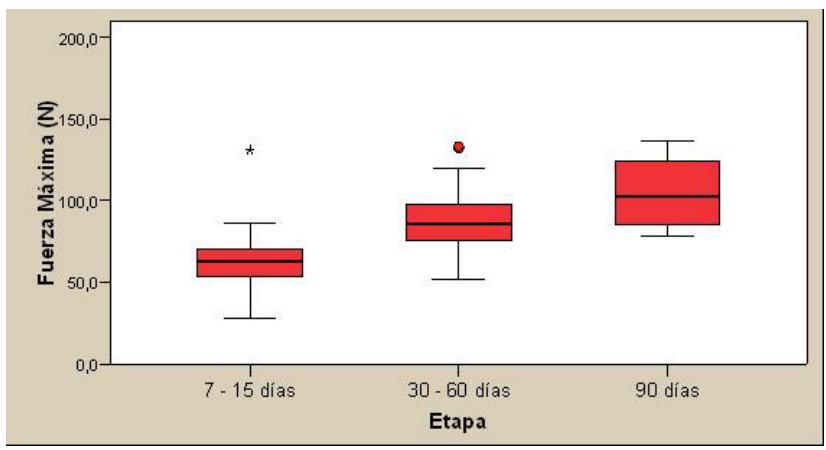

Fig. 2. Distintas etapas de tratamiento con respecto a la Fuerza Máxima (N).

entre drogas no resultaron estadísticamente significativas ( $p>0.05$ ), pero cabe mencionar que $(0)$ registró los valores medios de rigidez más bajos en todas las etapas. Tampoco se verificó interacción significativa entre los factores droga y etapa ( $p>0.05)$.

Fuerza Máxima: Los valores de más elevados de fuerza máxima aplicada, se registraron a los 90 días, y un tanto menos a los 60 días. En la Figura 2, se expresan las medias de cada etapa, agrupados en subconjuntos homogéneos. Dentro del subconjunto 1 , correspondiente a las primeras 3 etapas $(7,15$ y 30 días), las diferencias entre ellas no alcanzaron a ser significativas, pese al progresivo incremento que se observa en el tiempo $(p>0,05)$, pero ya a los 60 días, las diferencias se tornaron significativas $(p<0,05)$ respecto a las etapas anteriores. En tanto que el subconjunto 2 (etapas 30,60 y 90 días), los valores tienden a estabilizarse, con diferencias no significativas entre ellos.

Fuerza de Ruptura o Fractura: Los resultados son semejantes a la fuerza máxima (Fig. 3). 
Rigidez flexional: El aumento de rigidez un entre la primera y segunda etapa fue significativo, pero este incremento fue no significativo entre las etapas posteriores evaluadas.

Ensayo de Compresión Fuerza de Ruptura: Los valores de fuerza de ruptura en ensayos de compresión fueron similares en las tres etapas evaluadas, con una alta dispersión de valores, los cuales se reflejan en Fig. 4, al observar la desviación estándar (DE) y el rango de datos (Máx-Mín) calculado para cada droga y etapa. Pese a que las diferencias entre drogas no resultaron significativas, se puede mencionar que (PA), registró los valores medios más elevados en todas las etapas.

Rigidez flexional: De acuerdo al resultado del test de ANOVA, ningún factor (etapa o droga) influye significativamente sobre los valores de rigidez $(p>0,05)$. Tampoco se verificó interacción significativa entre los factores Droga y etapa $(p>0,05)$.El mismo comportamiento presento Tenacidad o energía absorbida $(\mathrm{mJ})$ y Fuerza Máxima $(\mathrm{N})$.

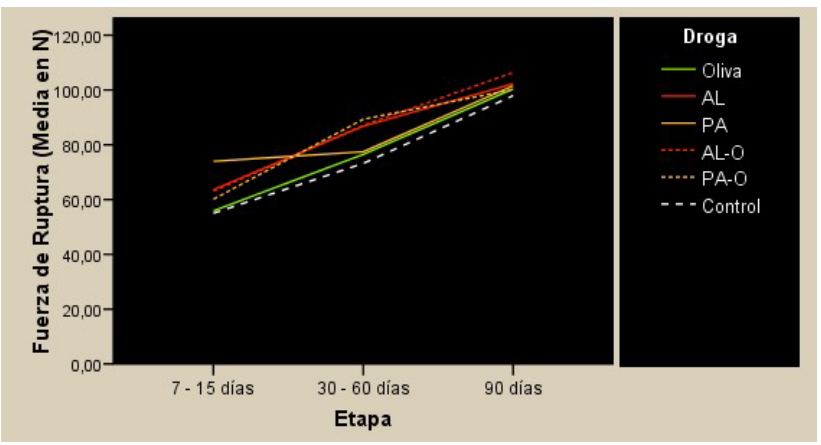

Fig. 3. Comportamiento de las drogas en las distintas etapas con respecto a la Fuerza de Ruptura.

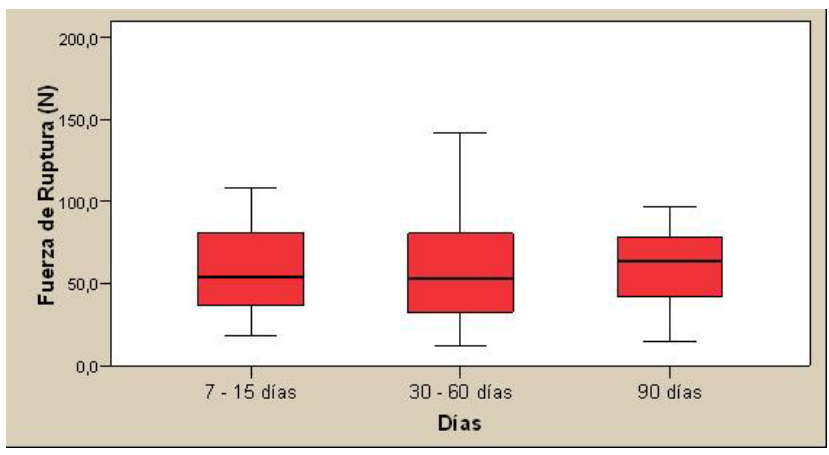

Fig. 4. Muestra las distintas etapas de tratamiento con respecto a la Fuerza de Ruptura (N).

\section{DISCUSIÓN}

El grosor y el diámetro del hueso cortical son los principales factores que afectan a su biomecánica. Un aumento de cualquiera de estas características provoca un aumento de la resistencia ósea. Un hueso largo se puede modelar como un cuerpo cilíndrico, y según las leyes básicas de la mecánica la resistencia a la deformación de cualquier cuerpo cilíndrico sometido a una fuerza es directamente proporcional a su diámetro. Por otro lado, el grosor de la región cortical y la cantidad de masa ósea están estrechamente relacionados, de manera que, con masa ósea constante, una variación en su distribución modifica también la resistencia del hueso. La disminución del grosor cortical que se produce con la edad o en algunas enfermedades osteodegenerativas lleva asociado un aumento del riesgo de fractura (Guede et al.).

Los modelos animales, en particular ratas, ofrecen la posibilidad de lograr naturalmente o la modificación genética de un fenotipo esquelético asociada con la enfermedad y la realización de ensayos de fractura destructivos en el hueso para determinar el cambio resultante en la mecánica del hueso propiedades. Por desgracia, el repertorio de pruebas mecánicas, disponibles para pequeños huesos de animales, es limitado debido a las limitaciones inherentes impuestas por el tamaño del hueso (Hang \& Barber, 2011).

En los últimos años, sin embargo, ha habido una explosión en el tipo de ensayos mecánicos propuestos para los huesos de ratón. Y debido a que el hueso del ratón ha jerarquía de la organización, estas bien definido ensayos de la escala de longitud natural de los niveles de minerales y proteínas para pruebas óseas. Por ejemplo nano-y las pruebas de microindentación se han realizado sobre bases consanguínea o genética huesos de ratón en el modo monótono o cíclico para determinar el local de viscoelástica, elástica y propiedades asociadas con minerales óseos y proteínas modificaciones (Hernández-Flores et al., 2011).

Para valorar el comportamiento biomecánico del hueso existen diferentes tipos de ensayos (tracción, compresión, torsión, flexión a tres y cuatro puntos), pero el ensayo de flexión a tres puntos es elmás adecuado para animales pequeños. Aunque el comportamiento mecánico del hueso es complejo, se ha simplificado de diferentes maneras con la finalidad de proponer modelos que se aproximen a su comportamiento "in vivo". 
Las propiedades biomecánicas del hueso, no son valores homogéneos que se puedan definir de forma precisa, ello depende de la distribución mineral, características estructurales, variaciones entre individuos y la función del hueso a estudiar (Manjulabala et al., 2009).

Los huesos largos son importantes porque se componen de hueso cortical puro y están cubiertos de periostio, pudiendo reaccionar por aposición perióstica como el hueso humano. Además el fémur de rata tiene la ventaja, como el del ser humano, de ser gran portador de cargas, por lo cual se lo puede someter a análisis con fuerzas más pesadas. Este modelo en fémur de rata Wistar es económico, de fácil manipulación, bajo costo de mantenimiento. Se pretendió analizar en este tipo de hueso largo los posibles efectos sistémicos que tuvieran las drogas, para prevenir efectos adversos en futuros estudios clínicos. Los ensayos biomecánicos en el fémur se realizaron previa limpieza e hidratación de las muestras con solución fisiológica y colocada en freezer a $-20^{\circ} \mathrm{C}$ para su conservación (Nazarian et al., 2008).

La mecánica y la ciencia de materiales estudian los efectos y la relación entre las fuerzas aplicadas sobre una estructura o cuerpo rígido y la deformación producida. El hueso, para su estudio, se puede considerar tanto un tejido como una estructura, ya que desempeña dos funciones básicas: control del metabolismo de $\mathrm{Ca}, \mathrm{P}$ y Mg (función fisiológica) y soporte del organismo y protección de órganos (función mecánica). La complejidad mecánica del tejido óseo, compuesto de hueso cortical y hueso trabecular, ambos con comportamientos mecánicos distintos, supera la de la mayoría de los materiales utilizados en ingeniería.

Los valores de más elevados de fuerza máxima aplicada y fuerza de fractura, se registraron a los 60 días, y un tanto menos a los 90 días. Las diferencias entre etapas resultaron significativas, verificándose que a los 7,15 y 30 días, las diferencias entre ellas no alcanzaron a ser significativas, pese al progresivo incremento que se observa en el tiempo ( $p>0,05)$, pero ya a los 60 días, las diferencias se tornaron significativas $(p<0,05)$ respecto a las etapas anteriores.

En cuanto a la energía absorbida las diferencias entre drogas no resultaron estadísticamente significativas al igual que las diferencias entre patas $(p>0,05)$. Tampoco se verificó interacción significativa entre los factores $(p>0,05)$. En cuanto al comportamiento del grupo $(\mathrm{O})$ se puede observar cambios en el comportamiento del hueso en comparación con las drogas administradas. Datos que se correlacionan con las diferentes variables analizadas. Lo que permite inferir que el (O) bajo estudio tiene un efecto significativo sobre la calidad ósea. Futuras investigaciones nos permitirán profundizar sobre el tema.

MacNamara et al. (2010) prueba la hipótesis de que las propiedades mecánicas del tejido trabecular ósea serán diferentes para los huesos normales, ovariectomizados y tratados con el fármaco de rata en el transcurso del envejecimiento. Estos resultados indican que la ovariectomía en ratas aumenta la rigidez, resistencia a la influencia, cepa rendimiento y la tensión de rotura del tejido mineralizado que constituye trabecular del hueso en relación a la normalidad.

Kolodziej et al. (1998) demostró que las altas concentraciones de PA en el hueso intacto disminuyeron la densidad mineral ósea y debilitan la resistencia mecánica de los fémures de rata. La resistencia mecánica del callo de cicatrización temprana no se correlacionó con la concentración de PA en el hueso.

Si bien se demostró que no hubo sinergia en el tratamiento combinado de estas drogas, es necesario seguir con investigaciones, para así poder combinar estas drogas en tiempos diferentes y su vez combinar bifosfonatos con otras drogas, para poder ser utilizadas en regeneración ósea y patologías de la cavidad bucal. Este tipo de investigaciones abrirán aún más el horizonte en el diseño de nuevos sistemas de fármacos para así poder incorporarlos al arsenal terapéutico en la práctica odontológica y sus distintas especialidades.

ESCUDERO, C.; VIRGA, C.; AGUZZI, A.; ARAMBURU, G. \& DE LEONARDI, A. Biomechanical evaluation of the effect of bisphosphonates and olive oil in a model of bone remodeling in rat femur. Effects on bone remodeling. Int. J. Odontostomat., 11(1):19-24, 2017.

SUMMARY: Studies have shown that olive oil $(O)$ with its phenolic compounds have positive effects on various physiological biomarkers. Previous analyzes have shown that bisphosphonates are potent inhibitors of bone resorption. The objective of this work was to study the effect of combined treatment with alendronate $(A L)$ and pamidronate $(P A)$ and $\mathrm{O}$ on bone regeneration. Formulas $0.5 \mathrm{mg} / \mathrm{kg}$ for $\mathrm{AL}$ dosed, and $0.6 \mathrm{mg} / \mathrm{kg}$ for PA. O was administered in the diet, $50 \mathrm{~g} /$ $\mathrm{kg}$. Fifty-four male Wistar rats were divided into 6 groups. The control group $(C)$ received weekly $0.3 \mathrm{~mL} / 100 \mathrm{~g}$ of saline subcutaneously. Group $(A L)$ received weekly subcutaneously in the left posterior limb. Group (PA) was placed as the 
ESCUDERO, C.; VIRGA, C.; AGUZZI, A.; ARAMBURU, G. \& DE LEONARDI, A. Evaluación biomecánica del efecto de bisfosfonatos y aceite de oliva en un modelo de remodelación óseo en fémur de rata. Efectos en la remodelación ósea. Int. J. Odontostomat., 11(1):19-24, 2017.

previous group. Group $(\mathrm{O})$ was treated in food and in the areas of surgery received subcutaneous injection with saline. The (ALO) group received combined treatment with $\mathrm{Al}$ and $O$. The group (PAO) was treated the same as before. Femur samples at times 15, 30, 60 and 90 days, were included in physiological solution and maintained at $-20{ }^{\circ} \mathrm{C}$ were obtained. Statistical studies were conducted through analysis of variance to two and three classification criteria. The ANOVA showed that only days factor significantly influences the values of the variables $(p<0.05)$. The differences between drugs were not statistically significant $(p>0.05)$. Nor was there significant drug interaction between factors and stage ( $p>$ $0.05)$ was verified. The highest values of force rupture applied occurred at 90 days. No significant differences were found in the biomechanical testing, demonstrating the systemic action of drugs. These actions were beneficial to increase rigidity.

KEY WORDS: bisphosphonates, olive oil, biomechanical testing.

\section{REFERENCIAS BIBLIOGRÁFICAS}

Buehler, M. J. Hierarchical Nanomechanics of Collagen Fibrils: Atomistic and Molecular Modeling. In: Fratzl, P. (Ed.). Collagen. Structure and Mechanics. New York, Springer, 2008.

Cicerale, S.; Lucas, L. \& Keast, R. Biological activities of phenolic compounds present in virgin olive oil. Int. J. Mol. Sci., 11(2):45879, 2010

Eppell, S. J.; Smith, B. N.; Kahn, H. \& Ballarini, R. Nano measurements with micro-devices: mechanical properties of hydrated collagen fibrils. J. R. Soc. Interface, 3(6):117-21, 2006.

Fuchs, R. K.; Shea, M.; Durski, S. L.; Winters-Stone, K. M.; Widrick, J. \& Snow, C. M. Individual and combined effects of exercise and alendronate on bone mass and strength in ovariectomized rats. Bone, 41(2):290-6, 2007.

Giavaresi, G.; Fini, M.; Gnudi, S.; Aldini, N. N.; Rocca, M.; Carpi, A. \& Giardino, R. Comparison of calcitonin, alendronate and fluorophosphate effects on ovariectomized rat bone. Biomed. Pharmacother., 55(7):397-403, 2001.

Guede, D.; Dapía, S. \& Caeiro, J. R. Relación entre las propiedades biomecánicas y las variaciones locales en la microestructura ósea en cabeza femoral humana osteoporótica. Rev. Osteoporos. Metab. Miner., 2(3):11, 2010.

Hang, F. \& Barber, A. H. Nano-mechanical properties of individual mineralized collagen fibrils from bone tissue. J. R. Soc. Interface, 8(57):500-5, 2011.

Hernández-Flores, C.; Delgado, A. \& Domínguez-Hernández, V. M. Evaluación biomecánica de un modelo de defecto óseo en tibia de rata. Rev. Mex. Ing. Biomed., 22(1):12-9, 2011.

MacNamara, A.; Button, A. \& Collins, D. The role of psychological characteristics in facilitating the pathway to elite performance Part 1: Identifying mental skills and behaviors. Sport Psychol., 24(1):52-73, 2010.

Madrona, A.; Pereira-Caro, G.; Mateos, R.; Rodríguez, G.; Trujillo, M.; Fernández-Bolaños, J. \& Espartero, J. L. Synthesis of hydroxytyrosyl alkyl ethers from olive oil waste waters. Molecules, 14(5):1762-72, 2009.

Manjulabala, I.; Lui, Y.; Epari, D. R.; Roschger, P.; Shell, H.; Fratzl, P. \& Duda, G. N. Spatial and temporal variations of mechanical properties and mineral content of the external callus during bone healing. Bone, 45(2):185-92, 2009.

Nazarian, A.; Bauernschmitt, M.; Eberle, C.; Meier, D.; Müller, R. \& Snyder, B. D. Design and validation of a testing system to assess torsional cancellous bone failure in conjunction with time-lapsed micro-computed tomographic imaging. J. Biomech., 41(16):3496501, 2008.

Kolodziej, P.; Lee, F. S.; Patel, A.; Kassab, S. S.; Shen, K. L.; Yang, K. H. \& Mast, J. W. Biomechanical evaluation of the schuhli nut. Clin. Orthop. Relat. Res., (347):79-85, 1998.

Dirección para correspondencia:

Alejandra Aguzzi

Cátedra de Farmacología y Terapéutica

Departamento de Patología Oral

Facultad de Odontología

Universidad Nacional de Córdoba

Cordoba

ARGENTINA

Email: aleceagu@yahoo.com.ar

Recibido : 13-07-2016

Aceptado: 23-12-2016 